Journal Club

Editor's Note: These short reviews of recent JNeurosci articles, written exclusively by students or postdoctoral fellows, summarize the important findings of the paper and provide additional insight and commentary. If the authors of the highlighted article have written a response to the Journal Club, the response can be found by viewing the Journal Club at www.jneurosci.org. For more information on the format, review process, and purpose of Journal Club articles, please see http://jneurosci.org/content/ preparing-manuscript\#journalclub.

\title{
CA2 Pyramidal Neurons: Biophysically and Anatomically Predisposed Integrators of Cortical Sensory Information
}

\author{
VViktor János Oláh ${ }^{1,3}$ and $\odot$ Miklos Szoboszlay ${ }^{2,3}$ \\ ${ }^{1}$ Laboratory of Cellular Neuropharmacology, ${ }^{2}$ Laboratory of Cellular Neurophysiology, Institute of Experimental Medicine, Hungarian Academy of \\ Sciences, H-1083 Budapest, Hungary, and 3János Szentágothai School of Neurosciences, Semmelweis University, H-1083 Budapest, Hungary \\ Review of Srinivas et al.
}

The hippocampus plays a key role in encoding, storing, and recalling memory, as well as processing spatiotemporal and sensory information (Buzsáki and Moser, 2013) The network topology that supports these sophisticated functions is traditionally summarized by the wiring diagram of the trisynaptic loop. This scheme is a simplified tripartite unidirectional circuit, where the dentate gyrus (DG) receives and processes the primary cortical input from the entorhinal cortex (EC) and its principal cells project to CA3 pyramidal cells (PCs). The CA3 PCs in turn excite CA1 PCs that are considered by the classical view as the primary output of the hippocampus. However, several recent discoveries in the largely neglected hippocampal CA2 region, which is interposed between the two larger and more intensely studied CA1 and CA3 regions, demand a substantial revision of the classical view (Jones and McHugh, 2011; Dudek et al., 2016). One of these publications, published in The Journal of Neuroscience by

Received May 23, 2017; revised July 8, 2017; accepted July 11, 2017.

This work was supported by the Kuffler Scholarship of the Stephen W. Kuffler Research Foundation and the Hungarian Brain Research Program Grant KTIA_13_NAP-A-I/5 to V.J.0. We thank Drs János Szabadics and Zoltan Nusser for their comments on the paper.

The authors declare no competing financial interests.

Correspondence should be addressed to Viktor János Oláh, Institute of

Experimental Medicine, Hungarian Academy of Sciences, H-1083 Budapest, Hungary. E-mail: olah.viktor@koki.mta.hu.

DOI:10.1523/JNEUROSCI.1405-17.2017

Copyright $\odot 2017$ the authors $\quad 0270-6474 / 17 / 377564-03 \$ 15.00 / 0$
Srinivas et al. (2017) revealed a subfieldspecific, several-fold stronger excitatory drive from the EC to CA2 PCs than to CA1 and CA3 PCs. The authors discovered that a delicate interplay between unique dendritic morphological features and physiological properties enables the stronger representation of EC activity in CA2 PCs. These findings contribute to the emerging hypothesis that the CA2 region is a potent relay station of processed cortical information, which potentially bypasses and complements the first stages of the trisynaptic loop.

The first aim of the study by Srinivas et al. (2017) was to understand what underlies an intriguing previous finding that stimulation of the distal EC fibers caused approximately fivefold larger somatic EPSPs in CA2 PCs than in neighboring CA1 PCs (Chevaleyre and Siegelbaum, 2010). Several factors can influence, either alone or in combination, the somatic readout of distal dendritic synaptic inputs. These include dendritic morphology, active and passive conductances, and quantal properties of the synapses (Magee, 1998; Andrasfalvy and Magee, 2001; London and Häusser, 2005). To investigate the specific involvement of these factors, Srinivas et al. (2017) first compared the amplitudes of unitary EPSPs elicited by glutamate uncaging on distal dendritic segments, a method that directly activates postsynaptic receptors within the photo- stimulated area without the involvement of presynaptic fibers. Surprisingly, this approach produced only twofold larger somatic EPSPs in CA2 versus CA1 PCs, which is a substantially smaller difference than the results of the previous EC fiber stimulation experiments. The authors showed that the differences in the EPSP amplitudes were not due to different spine morphology or AMPA receptor content in CA1 and CA2 PCs, which excludes disparate local depolarization as the underlying mechanism. Spine densities, however, were found to be different in the two cell types, as 3.5 times more spines were found in CA2 PC distal dendritic segments located in the termination zone of the EC inputs, the stratum lacunosum-moleculate (SLM), compared with CA1 PC distal dendrites. This observation suggests that the EC pathway forms several times more excitatory synapses with CA2 PCs than CA1 PCs.

Next, Srinivas et al. (2017) examined dendritic filtering in CA1 and CA2 PCs. The substantial contribution of hyperpolarization-activated nonselective cation channels (HCNs) to attenuation of distal dendritic inputs in CA1 PCs is well characterized (Magee, 1998; Lörincz et al., 2002). However, little is known about the subcellular distribution of these channels in CA2 PCs. Using immunohistochemical labeling, Srinivas et al. (2017) showed that the amount of $\mathrm{HCN} 1$ and $\mathrm{HCN} 2$ proteins in the strata radiatum (SR) and SLM was 
lower in CA2 than in CA1. These findings were supported by uncaging experiments, which showed that pharmacological blockade of HCN channels did not significantly alter the distally evoked responses in CA2 PCs, unlike in CA1 PCs. Dendritic filtering also depends on passive electrical properties and dendritic geometry (Stuart and Spruston, 1998). Srinivas et al. (2017) rigorously examined dendritic morphology in the two PC populations and found that although CA2 PCs had more dendritic bifurcations, and wider spread of dendritic branches in the SLM than CA1 PCs and their apical dendrites divided into secondary dendrites close to the soma, CA2 PCs formed fewer oblique dendrites in the SR. These morphological features allow CA2 PCs to receive more input from the EC, compared with CA1 PCs, and also support efficient dendro-somatic propagation (Sun et al., 2014) of these inputs via reduced branching in the $S R$, because the amount of dendritic bifurcations is inversely correlated with the efficiency of dendritic signal propagation. Together, these results suggest that CA2 PCs possess both morphological and physiological properties that enable robust somatic representation of distal dendritic inputs.

Despite the inevitable simplifications, sufficiently well constrained computational models can reinforce experimental results, as well as providing insights that cannot be easily gleaned from experiments. Therefore, Srinivas et al. (2017) built multicompartmental models of CA1 and CA2 PCs with experimentally derived morphological and physiological features. These models were able to replicate the authors' experimental findings. Importantly, the modeling results also provided an explanation for the different somatic readout of distal dendritic inputs (either a fivefold or twofold difference between the EPSPs evoked in CA1 or CA2 PCs by Chevaleyre and Siegelbaum, 2010 and Srinivas et al., 2017). According to the model, the fivefold EPSP difference originates from the combination of the distinct biophysical properties of CA1 and CA2 PCs (which explains only a twofold difference) and the different number of stimulated fibers. The latter difference accounts for the additional threefold difference in the EPSPs, which is not present if the EPSPs are tested using photostimulation of single spines in the SLM. Because the larger spine density in the distal dendrites of CA2 PCs compared with CA1 PCs suggests larger number of excitatory synapses from the EC to CA2 PCs, the same electrical stimulation in the SLM recruits more excitatory syn- apses, resulting in much larger EPSPs in CA2 PCs than in CA1 PCs. Overall, the modeling results strengthen the experimental conclusions that distinct dendritic morphologies, spine densities, and ion channel distributions are sufficient to account for the observed stronger effect of EC-EPSPs in CA2 PCs.

What are the potential functional consequences of the distinct cellular properties of CA2 PCs? Within the classical trisynaptic loop, the CA1 region is considered to be the main output of the hippocampus, and its activity primarily reflects inputs arriving from the EC and the CA3 (Bittner et al., 2015). However, it has recently been suggested that the CA2 region also conveys strong excitation onto CA1 PCs (Chevaleyre and Siegelbaum, 2010). The observations of Srinivas et al. (2017) add further essential details about the impact and potential roles of the CA2-CA1 pathway. Regarding the context of these discoveries, some fundamental in vivo findings have to be taken into consideration as well. Namely, in the absence of Schaffer collateral input from CA3 to CA1, CA1 PCs maintain high firing rates and spatially modulated activity patterns (Nakashiba et al., 2008). This suggests that the CA1 region can sufficiently perform complex network functions independent of CA3 activity, which has been shown to be one of the main excitatory drivers of CA1 PCs. Furthermore, because the distal EC inputs are not sufficient for driving CA1 cells (Takahashi and Magee, 2009), other strong afferents are needed for activating CA1 PCs while also conveying spatial information. The CA2 is an ideal candidate for this function due to the fact that the activity of CA2 PCs are spatially modulated (Mankin et al., 2015), similarly to the other CA regions. Together with the findings of Srinivas et al. (2017) this suggests that processed sensory information can effectively flow through the EC-CA2CA1 tripartite system, bypassing the conventional trisynaptic loop. This proposition gains further support from a recent publication (Boehringer et al., 2017) demonstrating that activation of CA2 efficiently silences the efferent impact of CA3 on CA1 via feedforward inhibition, which specifically limits excitation through the DG-CA3-CA1 channel. Recent publications therefore suggest that cortical information can enter the hippocampus through the CA2 region via a dedicated mechanism for effective representation of EC activity in CA2 PCs.

Although it has been established that EC inputs can potently recruit CA2 PCs, these cells also receive mossy fiber and Schaffer collateral inputs onto their more proximal dendritic regions. These proximal inputs appear to be ideal for driving CA2 PCs because they are less attenuated by dendritic filtering. Therefore, the comparison of the effects of proximal and distal excitatory inputs to CA2 PCs is essential to understand the potential functions of the EC-CA2 pathway. The Schaffer collaterals from CA3 PCs have been shown to evoke robust feedforward inhibition in CA2 PCs (Chevaleyre and Siegelbaum, 2010), which strongly limits the efficacy of this excitatory pathway, and synapses made by Schaffer collaterals have also been shown to be remarkably plasticityresistant (Zhao et al., 2007). The effect of the recently discovered mossy fiber input from DG granule cells to CA2 PCs (Kohara et al., 2014) is also limited by the miniscule size of the postsynaptic responses in CA2 PCs compared with those of the classical detonator synapses on CA3 PCs. In addition, these fibers originate from one of the most quiescence cells in the nervous system (Pernía-Andrade and Jonas, 2014). Thus, it is reasonable to assume that the EC input is one of the major, if not the most important determinant of CA2 PC activity. This idea is supported by the unique spine density, dendritic geometry, and active and passive electrical properties of CA2 PCs, as well as the fact that EC cells show remarkably high basal activity, regularly displaying high-frequency bursts in vivo (Burgalossi et al., 2011).

In conclusion, the wiring properties of the CA2 area suggests that its major function might be the direct routing of processed cortical sensory information to CA1 by bypassing the first two elements of the trisynaptic loop. This property of the system might be beneficial in some pathophysiological states that disrupt information flow from the CA3 to CA1. Although further studies are needed to confirm this new hypothesis, the findings of Srinivas et al. (2017) together with other recent studies on the CA2 region finally invite the smallest hippocampal region to join its larger neighbors at an eminent position within hippocampal circuit diagram.

\section{References}

Andrasfalvy BK, Magee JC (2001) Distancedependent increase in AMPA receptor number in the dendrites of adult hippocampal CA1 pyramidal neurons. J Neurosci 21:9151-9159. Medline

Bittner KC, Grienberger C, Vaidya SP, Milstein AD, Macklin JJ, Suh J, Tonegawa S, Magee JC (2015) Conjunctive input processing drives 
feature selectivity in hippocampal CA1 neurons. Nat Neurosci 18:1133-1142. CrossRef Medline

Boehringer R, Polygalov D, Huang AJY, Middleton SJ, Robert V, Wintzer ME, Piskorowski RA, Chevaleyre V, McHugh TJ (2017) Chronic loss of CA2 transmission leads to hippocampal hyperexcitability. Neuron 94:642-655.e9. CrossRef Medline

Burgalossi A, Herfst L, von Heimendahl M, Förste H, Haskic K, Schmidt M, Brecht M (2011) Microcircuits of functionally identified neurons in the rat medial entorhinal cortex. Neuron 70:773-786. CrossRef Medline

Buzsáki G, Moser EI (2013) Memory, navigation and theta rhythm in the hippocampalentorhinal system. Nat Neurosci 16:130-138. CrossRef Medline

Chevaleyre V, Siegelbaum SA (2010) Strong CA2 pyramidal neuron synapses define a powerful disynaptic cortico-hippocampal loop. Neuron 66:560-572. CrossRef Medline

Dudek SM, Alexander GM, Farris S (2016) Rediscovering area CA2: unique properties and functions. Nat Rev Neurosci 17:89-102. CrossRef Medline

Jones MW, McHugh TJ (2011) Updating hippocampal representations: CA2 joins the cir- cuit. Trends Neurosci 34:526-535. CrossRef Medline

Kohara K, Pignatelli M, Rivest AJ, Jung HY, Kitamura T, Suh J, Frank D, Kajikawa K, Mise N, Obata Y, Wickersham IR, Tonegawa S (2014) Cell type-specific genetic and optogenetic tools reveal hippocampal CA2 circuits. Nat Neurosci 17:269-279. CrossRef Medline

London M, Häusser M (2005) Dendritic computation. Annu Rev Neurosci 28:503-532. CrossRef Medline

Lörincz A, Notomi T, Tamás G, Shigemoto R, Nusser Z (2002) Polarized and compartmentdependent distribution of $\mathrm{HCN} 1$ in pyramidal cell dendrites. Nat Neurosci 5:1185-1193. CrossRef Medline

Magee JC (1998) Dendritic hyperpolarizationactivated currents modify the integrative properties of hippocampal CA1 pyramidal neurons. J Neurosci 18:7613-7624. Medline

Mankin EA, Diehl GW, Sparks FT, Leutgeb S, Leutgeb JK (2015) Hippocampal CA2 activity patterns change over time to a larger extent than between spatial contexts. Neuron 85 : 190-201. CrossRef Medline

Nakashiba T, Young JZ, McHugh TJ, Buhl DL, Tonegawa S (2008) Transgenic inhibition of synaptic transmission reveals role of CA3 output in hippocampal learning. Science 319: 1260-1264. CrossRef Medline

Pernía-Andrade AJ, Jonas P (2014) Theta-gammamodulated synaptic currents in hippocampal granule cells in vivo define a mechanism for network oscillations. Neuron 81:140-152. CrossRef Medline

Srinivas KV, Buss EW, Sun Q, Santoro B, Takahashi H, Nicholson DA, Siegelbaum SA (2017) The dendrites of CA2 and CA1 pyramidal neurons differentially regulate information flow in the cortico-hippocampal circuit. J Neurosci 37: 3276-3293. CrossRef Medline

Stuart G, Spruston N (1998) Determinants of voltage attenuation in neocortical pyramidal neuron dendrites. J Neurosci 18:3501-3510. Medline

Sun Q, Srinivas KV, Sotayo A, Siegelbaum SA (2014) Dendritic Na+ spikes enable cortical input to drive action potential output from hippocampal CA2 pyramidal neurons. Elife 3:e04551. CrossRef Medline

Takahashi H, Magee JC (2009) Pathway interactions and synaptic plasticity in the dendritic tuft regions of CA1 pyramidal neurons. Neuron 62:102-111. CrossRef Medline

Zhao M, Choi YS, Obrietan K, Dudek SM (2007) Synaptic plasticity (and the lack thereof) in hippocampal CA2 neurons. J Neurosci 27: 12025-12032. CrossRef Medline 